\title{
Numerical Simulation of Dynamic Fracturing using a Particle Method
}

\author{
Yuki IMAI ${ }^{1}$, Hitoshi MIKADA ${ }^{1}$, Tada-nori GOTO ${ }^{1}$ and Junichi TAKEKAWA \\ ${ }^{1}$ Dept. of Civil and Earth Res. Eng., Kyoto University
}

\begin{abstract}
Better understanding of failure mechanism of rocks benefits in many fields from rock engineering to earth sciences. Especially, it is essential to understand how fractures initiate and propagate under various loading conditions in order to clarify real rock fracture processes. For the interpretation of rock failure, many attempts have been made experimentally or using fracture mechanics theory. Although much of the knowledge available today is based on experimental observations and the theory successfully represented the propagation of predefined cracks, the failure mechanics are not fully understood by experimental results and it is difficult to describe the initiation and coalescence of cracks using the theory. Thus, in the recent years, numerical modeling, which may be less restrictive, has been applied to study crack behaviors, and we also approach to the rock failure based on numerical simulations. To represent rock failure, we use a Hamiltonian Particle method (HPM), one of particle methods. In the HPM, we do not need to use grids or meshes to discretize the rock model, and thus we could deal with the failure relatively easily. In spite of this advantage of the HPM, the applicability of the method to the failure phenomena have yet to be revealed fully. In the present study, we apply the HPM to rock failure under some different specimens and different loading conditions. As a result of our simulations, the HPM successfully reproduce failure pattern of brittle fracture observed rock fracture experiments and can observe micro cracks initiation and propagation. This suggests that the HPM is the useful tool to analyze rock failure.
\end{abstract}

\section{INTRODUCTION}

It is important to understand failure mechanism of rocks in many scientific and engineering fields. To analyze the behavior of rocks, many attempts have been made using fracture mechanics theorem. It is, however, difficult to describe the initiation and coalescence of cracks in rock mass using the theory. In recent years, some numerical approaches based on damage mechanics have been applied to represent the fracture behavior ${ }^{1), 2}$.

In the previous studies, the conventional numerical methods, like finite difference methods (FDM) and finite element methods (FEM), have often been used as simulators. On the other hand, particle methods are also used to represent the failure behavior of rocks ${ }^{3)}$. Since particle methods have some advantages over the conventional methods, it is important to verify the applicability of particle methods to the failure analyses of rock masses. Recently, a Hamiltonian particle method (HPM) was developed for elasto-dynamic problems by Suzuki and Koshizuka ${ }^{4}$. HPM was applied to seismic wave propagation simulations and indicated the effectiveness for representing the topology and refining the accuracy ${ }^{5)}$. In spite of this advantage of the HPM, the applicability of the method to the failure phenomena have yet to be revealed fully.

In the present study, we apply the HPM to rock failure under some different specimens and different loading conditions in order to verify the applicability to the failure behavior of rock masses.

\section{METHOD}

In the HPM, a continuum is represented as a set of particles and a motion of the continuum is approximated as a movement of the particles. Each particle has the weight function calculated by Eq. (1) and the deformation gradient tensor, $\mathbf{F}_{\mathrm{i}}$, is calculated using the weighted least squares, minimizing the error function $\mathrm{e}_{\mathrm{i}}$, in Eq. (2) on each particle:

$$
\begin{gathered}
w_{i j}= \begin{cases}1-\frac{\left|\mathbf{r}_{i j}^{0}\right|^{2}}{r_{e}^{2}} & \left|\mathbf{r}_{i j}^{0}\right|<r_{e} \\
0 & \left|\mathbf{r}_{i j}^{0}\right| \geq r_{e}\end{cases} \\
e_{i}=\sum_{j}\left|\mathbf{F}_{i} \mathbf{r}_{i j}^{0}-\mathbf{r}_{i j}\right|^{2} w_{i j}
\end{gathered}
$$

where $r_{\mathrm{ij}}^{0}$ and $\boldsymbol{r}_{\mathrm{ij}}$ are initial and current relative 
position vector between particle $i$ and $j$, respectively, and $r_{e}$ is the influence radius. Each particle only affected by the particles within the influence radius. The constitutive equation of elastic body is defined as

$$
\begin{aligned}
\boldsymbol{\varepsilon}_{i} & =\frac{1}{2}\left(\mathbf{F}_{i}^{T} \mathbf{F}_{i}-\mathbf{I}\right) \\
\boldsymbol{\sigma}_{i} & =2 \mu \boldsymbol{\varepsilon}_{i}+\lambda \operatorname{tr}\left(\boldsymbol{\varepsilon}_{i}\right) \mathbf{I}
\end{aligned}
$$

where $\boldsymbol{\varepsilon}_{\mathrm{i}}$ is the Green-Lagrange strain tensor, $\boldsymbol{\sigma}_{\mathrm{i}}$ is the first Piora-Kirchhoff stress tensor, and $\lambda$ and $\mu$ are the Lame constants. The elastic potential energy, $\mathrm{V}$, and the kinetic energy, $\mathrm{K}$, are expressed as

$$
\begin{aligned}
& V=\sum_{i} \frac{1}{2} \boldsymbol{\varepsilon}_{i}: \boldsymbol{\sigma}_{i} \Delta A_{i} \\
& K=\sum_{i} \frac{1}{2} m_{i}\left|\mathbf{v}_{i}\right|^{2}
\end{aligned}
$$

where $\Delta \mathrm{A}_{\mathrm{i}}, \mathrm{m}_{\mathrm{i}}$ and $\mathbf{v}_{\mathrm{i}}$ are the volume, mass and velocity vector of each particle, respectively. The total energy, Hamiltonian $\mathrm{H}$, is calculated as $\mathrm{H}=\mathrm{K}+\mathrm{V}$ and this equation leads the equation of motion of each particle expressed as:

$$
\begin{aligned}
& \rho \frac{\partial \mathbf{v}_{i}}{\partial t}=\sum_{j}\left(\mathbf{F}_{i} \boldsymbol{\sigma}_{i} \mathbf{A}_{i}^{-1} \mathbf{r}_{i j}^{0}+\mathbf{F}_{j} \boldsymbol{\sigma}_{j} \mathbf{A}_{j}^{-1} \mathbf{r}_{i j}^{0}\right) w_{i j} \\
& \mathbf{A}_{i}=\sum_{j} \mathbf{r}_{i j}^{0} \otimes \mathbf{r}_{i j}^{0} w_{i j}
\end{aligned}
$$

where $\rho$ is the bulk density.

In general, rock is a heterogeneous material and this heterogeneity causes failure of rock. Therefore, we consider the Weibull distribution as the basis for heterogeneity. The distribution function of the Weibull distribution is given by:

$$
F(t)=1-\exp \left\{-\left(\frac{t-\gamma}{\eta}\right)^{m}\right\}
$$

where $m$ is the shape parameter describing the scatter of $t, \eta$ is the scale parameter and $\gamma$ is the location parameter, which is the minimum value of $t$. The uniaxial compressive strength and the ratio of uniaxial tensile strength to uniaxial compressive strength of each particle distribute following this function. In this study, the shape parameter is 3 and the location parameter is 0 . For the uniaxial compressive and the ratio of uniaxial tensile strength to uniaxial compressive strength, the scale parameter are $200 \mathrm{MPa}$ and -0.1 , respectively.

A lot of shear failure criteria have been proposed for rock. Among these criteria, the Mohr-Coulomb criterion is widely used because of its simplicity. Therefore, we adopt this criterion for the shear failure. The Mohr-Coulomb criterion is expressed by:

$$
f_{\mathrm{c}}=\sigma_{1}-k_{\mathrm{f}} \sigma_{3}-\sigma_{\mathrm{c}}=0
$$

where $\sigma_{1}$ and $\sigma_{3}$ are the maximun and minimum stress, $\sigma_{\mathrm{c}}$ is the uniaxial compressive strength, $\mathrm{k}_{\mathrm{f}}=(1+\sin \phi)(1-\sin \phi)$, and $\phi$ is the friction angle. We calculate this function on each particle. Tensile failure occurs when the tensile stress acting on the rock reaches a critical value, which is the tensile strength. This criterion simply expressed as:

$$
f_{\mathrm{t}}=\sigma_{\mathrm{t}}-\sigma_{3}
$$

where $\sigma_{\mathrm{t}}$ is the uniaxial tensile strength of each particle and compression is positive in this study. After failure, each particle deforms in plastic condition and the stress of each particle is dropped according to magnitude of the minimum principal stress of each particle.

\section{NUMERICAL RESULTS}

We conduct Brazilian disk tests using the HPM. The parameters used in this study are shown in Table 1. Figure 1 shows the load-strain curve and the number of failure particles in the Brazilian disk test. In the first stage of loading, the axial load increases linearly. Around 0.1 millistrain, the number of failure particles increases gradually. The specimen has a non-linear behavior just before the macroscopic failure of the specimen. At the macroscopic failure, the number of failure particles increases and the axial loading decreases dramatically. Figure 2 shows the failure propagation in the Brazilian disk. In Figure 2(a), the tensile failures distribute in the center of the specimen.

Table 1: Parameters used in this study.

\begin{tabular}{ll}
\hline Identity & Value \\
\hline \hline Bulk density & $2,800 \mathrm{~kg} / \mathrm{m}^{3}$ \\
Deformation property & Young's modulus $60 \mathrm{GPa}$, Poisson's ratio 0.25 \\
& Friction angle $30^{\circ}$, Dilation angle $30^{\circ}$ \\
Strength property & Distribution of $\sigma_{c}: x_{0}=200 \mathrm{MPa}, x_{u}=0 \mathrm{MPa}, m_{w}=3$ \\
& Distribution of $\sigma_{t} / \sigma_{c}$ ratio: $x_{0}=-0.1, x_{u}=0 \mathrm{MPa}, m_{w}=3$ \\
Degradation property & $\delta_{c u}=95 \%, n_{d}=0.05$ \\
Particle arrangement & 501 particles in the diameter direction \\
& 196,321 particles in total \\
\hline
\end{tabular}




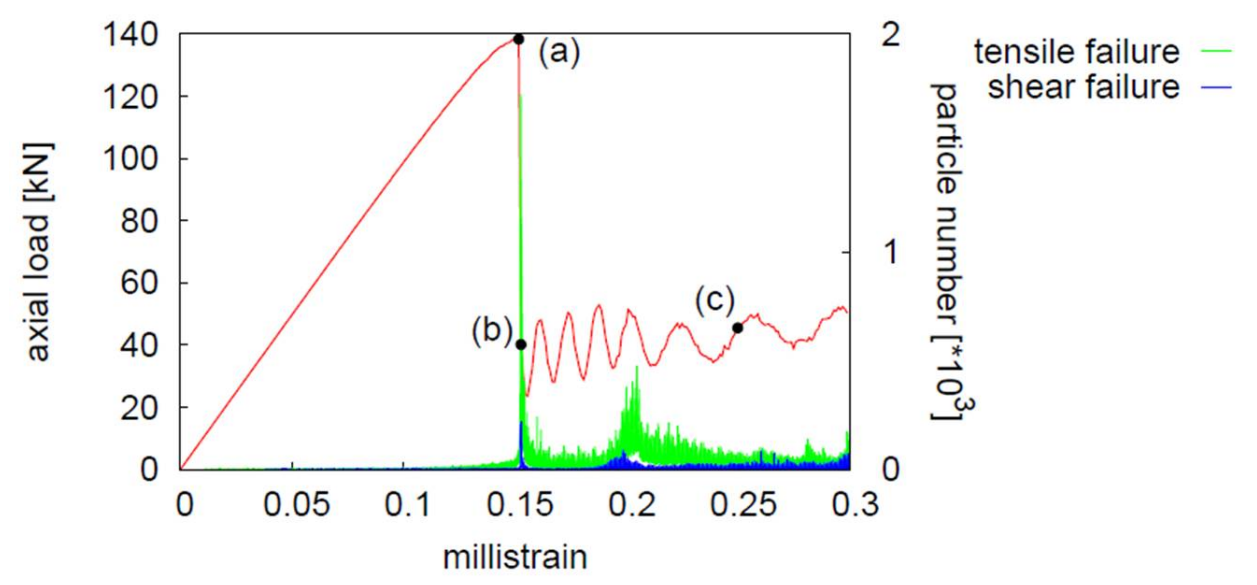

Figure 2: Load-strain curve and number of failure particles in 2D Brazilian disk test.

(a)

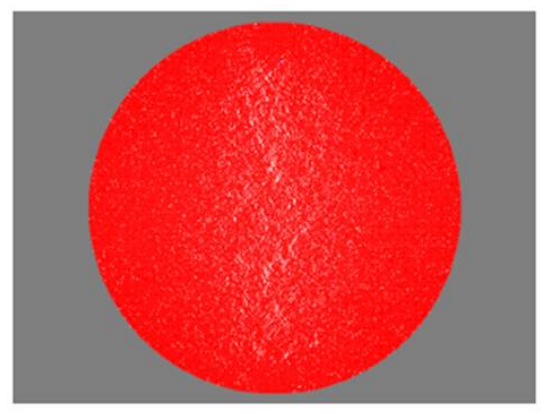

(b)

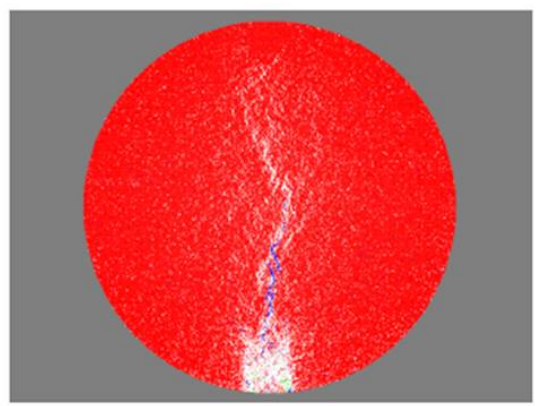

(c)

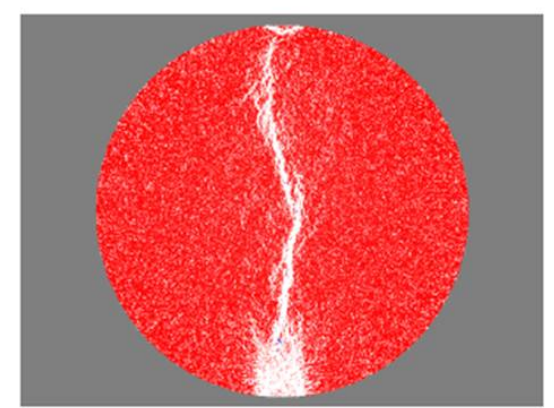

Figure 3: Macroscopic fracture propagation in 2D Brazilian disk test.

This indicates that the stress field in the specimen can be reproduced by the HPM accurately ${ }^{6}$. In Figure 2(b), the macroscopic failure forms in the center and the lower edge of the specimen. Finally, the macroscopic failure propagates upward and the specimen is separated by the macroscopic failure plane. These microscopic and macroscopic processes reproduced by our numerical simulation are similar to the results from the laboratory experiments.

\section{CONCLUSIONS}

We conducted the numerical Brazilian disk test using the HPM in order to verify the applicability of the HPM to the failure analyses of rock masses. Our numerical results, load-strain curve and microscopic and macroscopic failure behavior, showed good agreement with those from the laboratory experiments. We conclude that the HPM, which is one of the particle methods, can reproduce the failure behavior of rock masses.

\section{REFERENCES}

1) Fang, Z. and Harrison, J. P., 2002, Application of a local degradation model to the analysis of brittle fracture of laboratory scale rock specimens under triaxial conditions, Int. J. Rock. Mech. Min. Sci., 39, 443-457.

2) Tang, C.A., Liu, H.. Lee, P.K.K., Tsui, Y. and Tham, L.G., 2000, Numerical studies of the in uence of microstructure on rock failure in uniaxial compression - Part I: effect of heterogeneity, Int. J. Rock. Mech. Min. Sci., 37, 555-569.

3) Ma, G. W., Wang, X. J. and Ren, F., 2011, Numerical simulation of compressive failure of heterogeneous rock-like materials using SPH method, Int. J. Rock. Mech. Min. Sci., 48, 353-363.

4) Suzuki, Y. and Koshizuka, S., 2008, A Hamiltonian particle method for non-linear elastodynamics, Int. J. Numer. Methods Engng., 74, 1344-1373.

5) Takekawa, J., Madariaga, R., Mikada, H. and Goto, T., 2012, Numerical simulation of seismic wave propagation produced by earthquake by using a particle method, Geophys. J. Int., 191, 1305-1316.

6) Hondros, G., 1959, The evaluation of Poisson's ratio and the modulus of materials of a low tensile resistance by the Brazilian (indirect tensile) test with particular reference to concrete, Aust. J. Appl. Sci., 10, 234-264. 
